\title{
Alexander Polynomials of Knots Which Are Transformed into the Trefoil Knot by a Single Crossing Change
}

Dedicated to the memory of Yoko Nakagawa

YASUTAKA NAKANISHI

Department of Mathematics, Graduate School of Science, Kobe University, Rokko, Nada-ku, Kobe 657-8501, Japan

e-mail : nakanisi@math.kobe-u.ac.jp

Abstract. By the works of Kondo and Sakai, it is known that Alexander polynomials of knots which are transformed into the trivial knot by a single crossing change are characterized. In this note, we will characterize Alexander polynomials of knots which are transformed into the trefoil knot (and into the figure-eight knot) by a single crossing change.

\section{Introduction}

This note is a continuation of the previous work [4]. In the addendum, the author gave a necessary condition for Alexander polynomials of knots which are transformed into the trefoil knot by a single crossing change. By a refinement of this argument, we give a necessary and sufficient condition in this note.

Seifert [8] gave a characterization of the set of Alexander polynomials of all knots as the set of Laurent polynomials $f(t)$ with integral coefficients satisfying the following two conditions; (1) $f\left(t^{-1}\right)=f(t)$, and (2) $|f(1)|=1$.

A crossing change is a local move to exchange an over-crossing and an undercrossing between two knot diagrams $K_{1}$ and $K_{2}$ which are identical except near one point. Furthermore, we consider its spatial realization as follows: For two knots $k_{1}$ and $k_{2}$ represented by $K_{1}$ and $K_{2}, k_{1}$ and $k_{2}$ are said to be transformed into each other by a single crossing change.

Let $k$ be a knot, and $k^{\times}$the set of all knots obtained from $k$ by a single crossing change. Let $\Delta_{k}(t)$ be the Alexander polynomial of $k$, and $\Delta \mathcal{K}$ the set of the Alexander polynomials $\left\{\Delta_{k}(t)\right\}_{k \in \mathcal{K}}$ for a set of knots $\mathcal{K}$.

For the trivial knot $O$, Kondo [2] and Sakai [7] independently gave a characterization of $\Delta O^{\times}$as the set of Laurent polynomials $f(t)$ satisfying the following

Received August 18, 2009; revised January 27, 2012; accepted May 25, 2012.

2010 Mathematics Subject Classification: 57M25.

Key words and phrases: Alexander polynomials, Crossing change, Trefoil knot, Figureeight knot. 
two conditions; (1) $f\left(t^{-1}\right)=f(t)$, and (2) $|f(1)|=1$. In other words, there are no obstructions on Alexander polynomials for knots to be unknotting number one knots.

For the trefoil knot $3_{1}$, we give a characterization of $\Delta 3_{1}{ }^{\times}$as follows:

Theorem 1. Let $\zeta$ be a complex number such that $\zeta-1+\zeta^{-1}=0$. A Laurent polynomial $f(t)$ is contained in $\Delta 3_{1}{ }^{\times}$if and only if $f(t)$ satisfies the following three conditions; (1) $f\left(t^{-1}\right)=f(t)$, (2) $|f(1)|=1$, and (3) $f(\zeta)= \pm\left(a^{2}-a b+b^{2}\right)$ for some integers $a, b \in \mathbf{Z}$.

Remark 2. By a standard argument in Number Theory, Theorem 1 can be translated into the following. Let $\zeta$ be a complex number such that $\zeta-1+\zeta^{-1}=0$. A Laurent polynomial $f(t)$ is contained in $\Delta 3_{1} \times$ if and only if $f(t)$ satisfies the following three conditions; (1) $f\left(t^{-1}\right)=f(t),(2)|f(1)|=1$, and $(3) f(\zeta)=0, \pm 1$, or $\pm p_{1}^{e_{1}} \cdots p_{n}^{e_{n}}$ where $p_{i}$ 's are prime numbers, $e_{i}$ 's are even integers for $p_{i}=2,3 k+2$, and $e_{i}$ 's are arbitrary integers for $p_{i}=3,3 k+1$.

By the parallel argument, we give a similar result for the figure-eight knot $4_{1}$ as follows:

Theorem 3. Let $\zeta$ be a complex number such that $\zeta-3+\zeta^{-1}=0$. A Laurent polynomial $f(t)$ is contained in $\Delta 4_{1}{ }^{\times}$if and only if $f(t)$ satisfies the following three conditions; (1) $f\left(t^{-1}\right)=f(t)$, (2) $|f(1)|=1$, and $(3) f(\zeta)= \pm\left(a^{2}-3 a b+b^{2}\right)$ for some integers $a, b \in \mathbf{Z}$.

Remark 4. By a standard argument in Number Theory, Theorem 3 can be translated into the following. Let $\zeta$ be a complex number such that $\zeta-3+\zeta^{-1}=0$. A Laurent polynomial $f(t)$ is contained in $\Delta 4_{1} \times$ if and only if $f(t)$ satisfies the following three conditions; (1) $f\left(t^{-1}\right)=f(t)$, (2) $|f(1)|=1$, and $(3) f(\zeta)=0, \pm 1$, or $\pm p_{1}^{e_{1}} \cdots p_{n}^{e_{n}}$ where $p_{i}$ 's are prime numbers, $e_{i}$ 's are even integers for $p_{i}=2,3,5 k \pm 2$, and $e_{i}$ 's are arbitrary integers for $p_{i}=5,5 k \pm 1$.

The proofs of Theorems 1 and 3 are given in Section 3. The quick reviews for Remarks 2 and 4 are given in Section 4. In Section 2, we see the key fact of surgical description.

\section{Surgical description}

Let $k_{0}$ be a knot which is transformed into the trivial knot by a single crossing change. We assume that a knot $k$ is transformed into $k_{0}$ by a single crossing change. Then, $k$ is transformed into the trivial knot by twice crossing changes. By a surgical description of Alexander matrices, we can have the following.

Proposition 5. Let $k_{0}$ be a knot which is transformed into the trivial knot by a single crossing change. If a knot $k$ is transformed into $k_{0}$ by a single crossing change, then $k$ has an Alexander matrix $M(t)=\left(\begin{array}{cc}\Delta_{k_{0}}(t) & r\left(t^{-1}\right) \\ r(t) & m(t)\end{array}\right)$ of the following 
form:

(1) $\Delta_{k_{0}}(t)$ is the Alexander polynomial of $k_{0}$,

(2) $m\left(t^{-1}\right)=m(t)$ and $|m(1)|=1$, and

(3) $r(1)=0$.

On the other hand, for the matrix $M(t)$ satisfying the above conditions (1), (2), and (3), there exists a knot $k$ which is transformed into $k_{0}$ by a single crossing change such that $M(t)$ is an Alexander matrix of $k$.

We remark that the Alexander polynomial of a knot is determined by the determinant of Alexander matrices from a surgical view up to signs. The proof of Proposition 5 is essentially given in $[3,5,6]$, and also in the previous note [4]. The proof in [4] should need a correction. The readers should read " $m(t)=$ $1+\left(a_{2}+1\right)\left(\sqrt{t}-\frac{1}{\sqrt{t}}\right)^{2}+\cdots+\left(a_{2 n-2}+1\right)\left(\sqrt{t}-\frac{1}{\sqrt{t}}\right)^{2 n-2}+a_{2 n}\left(\sqrt{t}-\frac{1}{\sqrt{t}}\right)^{2 n} "$ as " $m(t)=1+\left(a_{2}+1\right)\left(\sqrt{t}-\frac{1}{\sqrt{t}}\right)^{2}+\cdots+(-1)^{n}\left(a_{2 n-2}+1\right)\left(\sqrt{t}-\frac{1}{\sqrt{t}}\right)^{2 n-2}+$ $(-1)^{n+1} a_{2 n}\left(\sqrt{t}-\frac{1}{\sqrt{t}}\right)^{2 n} "$ in the lines $-13--12$ in the page 331 .

\section{Proofs of theorems}

As the proofs of Theorems 1 and 3 are parallel, we give the proof of Theorem 1 mainly. We divide the proofs into two parts.

\subsection{Necessity part}

Let $k$ be a knot which is transformed into the trefoil knot by a single crossing change. From Proposition 5, the Alexander polynomial of $k, \Delta_{k}(t)$, is presented by the determinant up to signs: $\Delta_{k}(t)= \pm \operatorname{det}\left(\begin{array}{cc}\Delta_{3_{1}}(t) & r\left(t^{-1}\right) \\ r(t) & m(t)\end{array}\right)$ with $\Delta_{3_{1}}(t)=t-1+t^{-1}, m\left(t^{-1}\right)=m(t),|m(1)|=1$, and $r(1)=0$.

We take a complex number $\zeta$ such that $\Delta_{3_{1}}(\zeta)=\zeta-1+\zeta^{-1}=0$. Then, we have $\Delta_{k}(\zeta)= \pm\left(\Delta_{3_{1}}(\zeta) m(\zeta)-r(\zeta) r\left(\zeta^{-1}\right)\right)= \pm(\zeta) r\left(\zeta^{-1}\right)$. Let $r(t)=t^{p}\left(c_{0}+c_{1} t+\right.$ $\left.\cdots+c_{n-2} t^{n-2}+c_{n-1} t^{n-1}+c_{n} t^{n}\right)$ for some integers $c_{0}, c_{1}, \ldots, c_{n}$. Then, we have $r(\zeta)=\zeta^{p}\left(c_{0}+c_{1} \zeta+\cdots+c_{n-2} \zeta^{n-2}+c_{n-1} \zeta^{n-1}+c_{n} \zeta^{n}\right)=\zeta^{p}\left(c_{0}+c_{1} \zeta+\cdots+\left(c_{n-2}-\right.\right.$ $\left.\left.c_{n}\right) \zeta^{n-2}+\left(c_{n-1}+c_{n}\right) \zeta^{n-1}\right)=\cdots=\zeta^{p}(a-b \zeta)$ for some integers $a, b$.

Therefore, we have $\Delta_{k}(\zeta)= \pm r(\zeta) r\left(\zeta^{-1}\right)= \pm\left(a^{2}-a b+b^{2}\right)$.

In the case of the figure-eight knot, we take a complex number $\zeta$ such that $\Delta_{4_{1}}(\zeta)=\zeta-3+\zeta^{-1}=0$. Let $r(t)=t^{p}\left(c_{0}+c_{1} t+\cdots+c_{n-2} t^{n-2}+c_{n-1} t^{n-1}+c_{n} t^{n}\right)$ for some integers $c_{0}, c_{1}, \ldots, c_{n}$. Then, we have $r(\zeta)=\zeta^{p}\left(c_{0}+c_{1} \zeta+\cdots+c_{n-2} \zeta^{n-2}+\right.$ $\left.c_{n-1} \zeta^{n-1}+c_{n} \zeta^{n}\right)=\zeta^{p}\left(c_{0}+c_{1} \zeta+\cdots+\left(c_{n-2}-c_{n}\right) \zeta^{n-2}+\left(c_{n-1}+3 c_{n}\right) \zeta^{n-1}\right)=$ $\cdots=\zeta^{p}(a-b \zeta)$ for some integers $a, b$. Therefore, we have $\Delta_{k}(\zeta)= \pm r(\zeta) r\left(\zeta^{-1}\right)=$ $\pm\left(a^{2}-3 a b+b^{2}\right)$. 


\subsection{Sufficiency part}

First, we will see the degree trick that the following two lemmas are equivalent.

Lemma 6. Let $f(t)$ be a Laurent polynomial satisfying the following three conditions; (1) $f\left(t^{-1}\right)=f(t)$, (2) $|f(1)|=1$, and (3) $f(\zeta)= \pm\left(a^{2}-a b+b^{2}\right)$ for some integers $a, b$. Then, there exists a knot $k$ which is transformed into the trefoil knot by a single crossing change such that $\Delta_{k}(t)=f(t)$.

Lemma 7. Let $g(t)$ be a Laurent polynomial satisfying the following four conditions; (1) $g\left(t^{-1}\right)=g(t)$, (2) $|g(1)|=1$, (3) $g(\zeta)= \pm\left(a^{2}-a b+b^{2}\right)$ for some integers $a, b$, and (4) $g(t)=p t-(2 p-g(1))+p t^{-1}$. Then, there exists a knot $k$ which is transformed into the trefoil knot by a single crossing change such that $\Delta_{k}(t)=g(t)$.

It is clear that Lemma 6 implies Lemma 7.

We suppose that Lemma 7 holds. Let $f(t)$ be a Laurent polynomial satisfying the following three conditions; (1) $f\left(t^{-1}\right)=f(t),(2)|f(1)|=1$, and $(3) f(\zeta)=$ $\pm\left(a^{2}-a b+b^{2}\right)$ for some integers $a, b$. We take a Laurent polynomial $g(t)$ satisfying the following four conditions; (1) $g\left(t^{-1}\right)=g(t),(2) g(1)=f(1),(3) g(\zeta)=f(\zeta)$, and (4) $g(t)=p t-(2 p-g(1))+p t^{-1}$. From Proposition 5 , there exists a knot $l$ which is transformed into the trefoil knot by a single crossing change such that $\Delta_{l}(t)=g(t)$. Furthermore, $\Delta_{l}(t)= \pm \operatorname{det}\left(\begin{array}{cc}\Delta_{3_{1}}(t) & r\left(t^{-1}\right) \\ r(t) & m(t)\end{array}\right)=g(t)=p t-(2 p-g(1))+p t^{-1}$.

We take the Laurent polynomial $D(t)=g(t)-f(t)$. Then, $D\left(t^{-1}\right)=g\left(t^{-1}\right)-$ $f\left(t^{-1}\right)=g(t)-f(t)=D(t) . \quad D(1)=g(1)-f(1)=0 . \quad D(\zeta)=g(\zeta)-f(\zeta)=0$. Hence, we have $D(t)=(t-1)\left(t^{-1}-1\right) \Delta_{3_{1}}(t) d(t)$ for some Laurent polynomial $d(t)$ satisfying $d\left(t^{-1}\right)=d(t)$.

The matrix $M(t)=\left(\begin{array}{cc}\Delta_{3_{1}}(t) & r\left(t^{-1}\right) \\ r(t) & m(t) \mp(t-1)\left(t^{-1}-1\right) d(t)\end{array}\right)$ can be realized by an Alexander matrix of a knot which is transformed into the trefoil knot by a single crossing change from a surgical view.

$$
\pm \operatorname{det} M(t)= \pm \operatorname{det}\left(\begin{array}{cc}
\Delta_{3_{1}}(t) & r\left(t^{-1}\right) \\
r(t) & m(t) \mp(t-1)\left(t^{-1}-1\right) d(t)
\end{array}\right)= \pm\left(\Delta_{3_{1}}(t)(m(t) \mp\right.
$$

$\left.\left.(t-1)\left(t^{-1}-1\right) d(t)\right)-r(t) r\left(t^{-1}\right)\right)=g(t)-D(t)=f(t)$.

The degree trick is shown.

From now, we will give the proof of Lemma 7. It is sufficient to show the following Lemma 8. (If $g(1)=-1$, consider $-g(t)$.) That is the second trick.

Lemma 8. Let $g(t)$ be a Laurent polynomial satisfying the following four conditions; (1) $g\left(t^{-1}\right)=g(t)$, (2) $g(1)=1$, (3) $g(\zeta)=\varepsilon\left(a^{2}-a b+b^{2}\right)$ for some integers $a, b$ and $\varepsilon= \pm 1$, and (4) $g(t)=p t-(2 p-1)+p t^{-1}$. Then, there exists a knot $k$ which is transformed into the trefoil knot by a single crossing change such that $\Delta_{k}(t)=f(t)$.

Proof of Lemma 8. We remark that $g(\zeta)=p \zeta-(2 p-1)+p \zeta^{-1}=p-(2 p-1)=$ 
$-p+1=\varepsilon\left(a^{2}-a b+b^{2}\right)$ implies $p=1-\varepsilon\left(a^{2}-a b+b^{2}\right)$.

We consider the matrix $M(t)=\left(\begin{array}{cc}\Delta_{3_{1}}(t) & \left(t^{-1}-1\right)\left(a t^{-1}-b\right) \\ (t-1)(a t-b) & a b t-(2 a b+\varepsilon)+a b t^{-1}\end{array}\right)$, which satisfies the conditions (1), (2), and (3) in Proposition 5. Hence, there exists a knot $k$ which is transformed into the trefoil knot $3_{1}$ by a single crossing change such that $M(t)$ is an Alexander matrix of $k$. We calculate the Alexander polynomial: $-\varepsilon \operatorname{det} M(t)=-\varepsilon\left(\Delta_{3_{1}}(t)\left(a b t-(2 a b+\varepsilon)+a b t^{-1}\right)-(t-1)\left(t^{-1}-1\right)(a t-b)\left(a t^{-1}-b\right)\right)=$ $-\varepsilon\left(\left(-\varepsilon+a^{2}+b^{2}-a b\right) t+\left(\varepsilon-2 a^{2}-2 b^{2}+2 a b\right)+\left(-\varepsilon+a^{2}+b^{2}-a b\right) t t^{-1}\right)=\left(1-\varepsilon\left(a^{2}+\right.\right.$ $\left.\left.b^{2}-a b\right)\right) t+\left(-1+2 \varepsilon\left(a^{2}+b^{2}-a b\right)+\left(1-\varepsilon\left(a^{2}+b^{2}-a b\right)\right) t^{-1}=p t-(2 p-1)+p t^{-1}=g(t)\right.$. The proof of Lemma 8 is completed.

In the case of the figure-eight knot, we use $\Delta_{4_{1}}(t)=t-3+t^{-1}$ instead of $\Delta_{3_{1}}(t)=t-1+t^{-1}$.

The proofs of Theorems 1 and 3 are completed.

\section{Quick reviews for remarks}

By a standard argument in Number Theory, we can give the proofs of Remarks 2 and 4 . Here, we give quick reviews of the proofs for topologists who do not know enough much Number Theory as in compliance with the referee's suggestion.

It is sufficient to show the following propositions.

Proposition 9. An integer $N$ satisfies the condition $N= \pm\left(a^{2}-a b+b^{2}\right)$ for some integers $a, b \in \mathbf{Z}$ if and only if $N$ satisfies the condition $N=0, \pm 1$, or $\pm p_{1}^{e_{1}} \cdots p_{n}^{e_{n}}$ where $p_{i}$ 's are prime numbers, $e_{i}$ 's are even integers for $p_{i}=2,3 k+2$, and $e_{i}$ 's are arbitrary integers for $p_{i}=3,3 k+1$.

Proposition 10. An integer $N$ satisfies the condition $N= \pm\left(a^{2}-3 a b+b^{2}\right)$ for some integers $a, b \in \mathbf{Z}$ if and only if $N$ satisfies the condition $N=0, \pm 1$, or $\pm p_{1}^{e_{1}} \cdots p_{n}^{e_{n}}$ where $p_{i}$ 's are prime numbers, $e_{i}$ 's are even integers for $p_{i}=2,3,5 k \pm 2$, and $e_{i}$ 's are arbitrary integers for $p_{i}=5,5 k \pm 1$.

We can find an exact proof of Proposition 9 in a standard textbook of Number Theory by Takagi [9] (and also Ireland-Rosen [1]), in which we can also find a suggestive proof of Proposition 10.

\subsection{Sufficiency part for Proposition 9}

Let $J$ be the set of integers $N=a^{2}-a b+b^{2}$ for some integers $a, b \in \mathbf{Z}$.

Claim 11. Let $N_{1}, N_{2}$ be integers in J. Then, the product $N_{1} N_{2}$ is also in J.

Put $N_{1}=a^{2}-a b+b^{2}$ and $N_{2}=c^{2}-c d+d^{2}$. Then, we have $N_{1} N_{2}=$ $\left(a^{2}-a b+b^{2}\right)\left(c^{2}-c d+d^{2}\right)=(a c-b d)^{2}-(a c-b d)(a d+b c-b d)+(a d+b c-b d)^{2}$.

We take a complex number $\omega$ such that $\omega^{2}+\omega+1=0$. Let $\mathbf{Z}[\omega]$ be the ring $\{a+b \omega \mid a, b \in \mathbf{Z}\}$.

Claim 12. Let $p$ be a prime number. Then, we have one of the following: 
$p=a^{2}-a b+b^{2}$ for some integers $a, b \in \mathbf{Z}$, or (2) $p$ is prime in $\mathbf{Z}[\omega]$.

We remark that $\mathbf{Z}[\omega]$ is a Unique Factorization Domain (say U.F.D.), and we consider the unique prime factorization in $\mathbf{Z}[\omega]: p=\left(a_{1}+b_{1} \omega\right)\left(a_{2}+b_{2} \omega\right) \cdots\left(a_{k}+\right.$ $\left.b_{k} \omega\right)$ for some integers $a_{1}, b_{1}, \ldots, a_{k}, b_{k}$. Let $c+d \omega=\left(a_{2}+b_{2} \omega\right) \cdots\left(a_{k}+b_{k} \omega\right)$. Then, $p=\left(a_{1}+b_{1} \omega\right)(c+d \omega)=\left(a_{1} c-b_{1} d\right)+\left(a_{1} d+b_{1} c-b_{1} d\right) \omega \cdot a_{1} d+b_{1} c-b_{1} d=0$ implies $\left(a_{1}-b_{1}\right) d=-b_{1} c$ and $c+d \omega=m\left(\left(a_{1}-b_{1}\right)-b_{1} \omega\right)=m\left(a_{1}+b_{1} \omega^{2}\right)$ for some integer $m$. Then, we have $p=m\left(a_{1}+b_{1} \omega\right)\left(a_{1}+b_{1} \omega^{2}\right)=m\left(a_{1}^{2}-a_{1} b_{1}+b_{1}^{2}\right)$. Since $p$ is prime in $\mathbf{Z}$, we have $m=1$ or $a_{1}^{2}-a_{1} b_{1}+b_{1}^{2}=1$. The condition $m=1$ implies that $p=a^{2}-a b+b^{2}$ for some integers $a, b \in \mathbf{Z}$. The condition $a_{1}^{2}-a_{1} b_{1}+b_{1}^{2}=1$ implies that $p$ is prime in $\mathbf{Z}[\omega]$.

Claim 13. Let $p$ be a prime number such that $p \equiv 1(\bmod 3)$. Then, $p=a^{2}-a b+b^{2}$ for some integers $a, b \in \mathbf{Z}$.

This clam is the most difficult part in the review. We use the Legendre symbol in the standard argument to give the proof of Claim 13, which is rather long. Here, we give an alternate proof without it as follows:

Let $p$ be a prime number such that $p \equiv 1(\bmod 3)$. The group $F_{p}^{\times}$is a cyclic group of order $p-1=3 k$. There exists an element $a$ of order 3 in $F_{p}^{\times}$. Then, we have $a^{3}=1$ and $a^{3}-1=(a-1)\left(a^{2}+a+1\right)=0$. Since $a-1 \neq 0$ in $F_{p}$, we have $a^{2}+a+1=0$ in $F_{p}$. Hence, we have $a^{2}+a+1=p k$ in $\mathbf{Z}$ for some integer $k$. We consider the prime factorization of $(a-\omega)\left(a-\omega^{2}\right)=a^{2}+a+1=p k \in \mathbf{Z}[\omega]$. If $p$ was prime in $\mathbf{Z}[\omega]$, then $p$ would be a divisor of $a-\omega$ or $a-\omega^{2}$. It contradicts that $\frac{a-\omega}{p}, \frac{a-\omega^{2}}{p} \notin \mathbf{Z}[\omega]$. Therefore, $p$ is not prime in $\mathbf{Z}[\omega]$. By Claim 12, there exist some integers $a, b \in \mathbf{Z}$ such that $p=a^{2}-a b+b^{2}$.

Claim 14. Let $N$ be an integer such that $N=0,1$, or $p_{1}^{e_{1}} \cdots p_{n}^{e_{n}}$ where $p_{i}$ 's are prime numbers, $e_{i}$ 's are even integers for $p_{i}=2,3 k+2$, and $e_{i}$ 's are arbitrary integers for $p_{i}=3,3 k+1$, then $N=a^{2}-a b+b^{2}$ for some integers $a, b \in \mathbf{Z}$.

If $N=0$, then $N=a^{2}-a b+b^{2}$ for $a=b=0$. If $N=1$, then $N=a^{2}-a b+b^{2}$ for $a=b=1$. If $N=3$, then $N=a^{2}-a b+b^{2}$ for $a=1, b=-1$. For an integer $p$ such that $p=2,3 k+2, p^{2}=a^{2}-a b+b^{2}$ for $a=p, b=0$. For a prime integer $p$ such that $p=3 k+1, p=a^{2}-a b+b^{2}$ for some integers $a, b \in \mathbf{Z}$ by Claim 13. If $N=p_{1}^{e_{1}} \cdots p_{n}^{e_{n}}$ where $p_{i}$ 's are prime numbers, $e_{i}$ 's are even integers for $p_{i}=2,3 k+2$, and $e_{i}$ 's are arbitrary integers for $p_{i}=3,3 k+1$, then $N=a^{2}-a b+b^{2}$ for some integers $a, b \in \mathbf{Z}$ by Claim 11 . The sufficiency part is completed.

\subsection{Necessity part for Proposition 9}

Since $a^{2}-a b+b^{2}=(a-2 b)^{2}+3 a b \equiv 0,1(\bmod 3)$, we have the following:

Claim 15. Let $N$ be an integer in $J$. Then, we have $N \equiv 0,1(\bmod 3)$.

Since $\mathbf{Z}$ is a U.F.D., we consider the unique prime factorization in $\mathbf{Z}: a^{2}-a b+$ $b^{2}=p_{1}^{e_{1}} \cdots p_{n}^{e_{n}}$ where $p_{i}$ 's are mutually distinct prime numbers and $e_{i}$ 's are integers. 
If $p_{i}$ be an integer such that $p_{i} \equiv 2(\bmod 3)$, there do not exist integers $a, b$ such that $p_{i}=a^{2}-a b+b^{2}$ by Claim 15. By Claim 12, $p_{i}$ is prime in $\mathbf{Z}[\omega]$ and $e_{i}$ should be even. The necessity part is complete.

\subsection{Review for Proposition 10}

If $c=a-b, d=-b$, then $c^{2}+c d-d^{2}=(a-b)^{2}-(a-b) b-b^{2}=a^{2}-3 a b+b^{2}$. We have the following:

Claim 16. $\left\{N \mid N=a^{2}-3 a b+b^{2}\right\}=\left\{N \mid N=a^{2}+a b-b^{2}\right\}$.

It is sufficient to show Proposition 17 by Claim 16 .

Proposition 17. An integer $N$ satisfies the condition $N= \pm\left(a^{2}+a b-b^{2}\right)$ for some integers $a, b \in \mathbf{Z}$ if and only if $N$ satisfies the condition $N=0, \pm 1$, or $\pm p_{1}^{e_{1}} \cdots p_{n}^{e_{n}}$ where $p_{i}$ 's are prime numbers, $e_{i}$ 's are even integers for $p_{i}=2,3,5 k \pm 2$, and $e_{i}$ 's are arbitrary integers for $p_{i}=5,5 k \pm 1$.

The quick review for Proposition 17 is almost parallel to the above except for Claim 13 and we omit it here.

Acknowledgements The author would like to thank Professor Takashi Taniguchi for his suggestion for the interpretation of Theorems 1 and 3 as in Remarks 2 and 4. The author would also like to thank the referee for his careful reading and suggestion, especially for pointing out careless mistakes in Proof of Lemma 8.

\section{References}

[1] K. Ireland and M. Rosen, A Classical Introduction to Modern Number Theory, Graduate Texts Math., 84, Second Edition, Springer-Verlag, New York, 1990.

[2] H. Kondo, Knots of unknotting number 1 and their Alexander polynomials, Osaka J. Math., 16(1979), 551-559

[3] J. Levine, A characterization of knot polynomials, Topology, 4(1965), 135-141.

[4] Y. Nakanishi, Local moves and Gordian complexes, II, Kyungpook Math. J., 47(2007), 329-334.

[5] D. Rolfsen, A surgical view of Alexander's polynomial, in Geometric Topology (Proc. Park City, 1974), Lecture Notes in Math. 438, Springer-Verlag, Berlin and New York, 1974, pp. 415-423.

[6] D. Rolfsen, Knots and Links, Math. Lecture Series 7, Publish or Perish Inc., Berkeley, 1976.

[7] T. Sakai, A remark on the Alexander polynomials of knots, Math. Sem. Notes Kobe Univ., 5(1977), 451-456.

[8] H. Seifert, Über das Geschlecht von Knoten, Math. Ann., 110(1934), 571-592. 
[9] T. Takagi, Shotou Seisuuron Kougi (in Japanese) [Lectures on Elementary Number Theory], Second Edition, Kyoritsu Shuppan, Tokyo, 1971. 